Bull. Austral. Math. Soc.

47н09, 49м05

VOL. 57 (1998) [117-127]

\title{
APPROXIMATING COMMON FIXED POINTS OF TWO NONEXPANSIVE MAPPINGS IN BANACH SPACES
}

\section{Sachiko Atsushiba and Wataru Takahashi}

Let $C$ be a nonempty closed convex subset of a real Banach space $E$ and let $S, T$ be nonexpansive mappings of $C$ into itself. In this paper, we consider the following iteration procedure of Mann's type for approximating common fixed points of two mappings $S$ and $T$ :

$$
x_{1}=x \in C, x_{n+1}=\alpha_{n} x_{n}+\left(1-\alpha_{n}\right) \frac{1}{n^{2}} \sum_{i, j=0}^{n-1} S^{i} T^{j} x_{n} \text { for every } n \geqslant 1,
$$

where $\left\{\alpha_{n}\right\}$ is a sequence in $[0,1]$. Using some ideas in the nonlinear ergodic theory, we prove that the iterates converge weakly to a common fixed point of the nonexpansive mappings $T$ and $S$ in a uniformly convex Banach space which satisfies Opial's condition or whose norm is Fréchet differentiable.

\section{INTRODUCTION}

Let $C$ be a nonempty closed convex subset of a Banach space $E$. Then, a mapping $T: C \rightarrow C$ is called nonexpansive if $\|T x-T y\| \leqslant\|x-y\|$ for all $x, y \in C$. We denote by $F(T)$ the set of fixed points of $T$. Mann [5] introduced an iteration procedure for approximating fixed points of a mapping $T$ in a Hilbert space as follows:

$$
x_{1}=x \in C, x_{n+1}=\alpha_{n} x_{n}+\left(1-\alpha_{n}\right) T x_{n} \text { for every } n \geqslant 1,
$$

where $\left\{\alpha_{n}\right\}$ is a sequence in $[0,1]$. Later, Reich [7] discussed this iteration procedure in a uniformly convex Banach space whose norm is Fréchet differentiable.

In this paper, we consider the following iteration procedure of Mann's type for approximating common fixed points of two nonexpansive mappings in a Banach space:

$$
x_{1}=x \in C, x_{n+1}=\alpha_{n} x_{n}+\left(1-\alpha_{n}\right) \frac{1}{n^{2}} \sum_{i, j=0}^{n-1} S^{i} T^{j} x_{n} \quad \text { for every } n \geqslant 1,
$$

where $\left\{\alpha_{n}\right\}$ is a sequence in $[0,1]$ and $S, T$ are nonexpansive mappings of $C$ into itself. Using some ideas in the nonlinear ergodic theory and an inequality obtained by $\mathrm{Xu}[\mathbf{9}]$, we prove that the iterates converge weakly to a common fixed point of the two nonexpansive mappings in a uniformly convex Banach space which satisfies Opial's condition or whose norm is Fréchet differentiable.

Received 6th May, 1997

Copyright Clearance Centre, Inc. Serial-fee code: 0004-9729/98 \$A2.00+0.00. 


\section{Preliminaries}

Throughout this paper, we assume that $E$ is a real Banach space. We denote by $E^{*}$ the dual space of $E$ and also denote by $\left\langle y, x^{*}\right\rangle$ the value of $x^{*} \in E^{*}$ at $y \in E$. We write $x_{n} \rightarrow x$ (or $w$ - $\lim _{n \rightarrow \infty} x_{n}=x$ ) to indicate that the sequence $\left\{x_{n}\right\}$ of vectors converges weakly to $x$. Similarly $x_{n} \rightarrow x$ (or $\lim _{n \rightarrow \infty} x_{n}=x$ ) will symbolise strong convergence. We denote by $\mathbb{N}$ the set of all positive integers. For a subset $A$ of $E, \operatorname{co} A$ and $\overline{c o} A$ mean the convex hull of $A$ and the closure of the convex hull of $A$, respectively. We say that $E$ satisfies Opial's condition [6] if for any sequence $\left\{x_{n}\right\} \subset E$ with $x_{n} \rightarrow x \in E$, the inequality

$$
\liminf _{n \rightarrow \infty}\left\|x_{n}-x\right\|<\liminf _{n \rightarrow \infty}\left\|x_{n}-y\right\|
$$

holds for every $y \in E$ with $y \neq x$. It is known that all Hilbert spaces and $\ell^{p}$ with $1<p<\infty$ satisfy Opial's condition. It is also known that every separable Banach space can be equivalently renormed so that it satisfies Opial's condition (see [3]). We also know that if a Banach space $E$ has a duality mapping which is weakly sequentially continuous at 0 , then $E$ satisfies Opial's condition (see [4]). However, the spaces $L^{p}$ with $1<p<\infty$ and $p \neq 2$ do not satisfy Opial's condition (see also $[6]$ ). Let $E$ be a Banach space. Then, the norm of $E$ is said to be Gâteaux differentiable if

$$
\lim _{t \rightarrow 0} \frac{\|x+t y\|-\|x\|}{t}
$$

exists for each $x$ and $y$ in $S_{E}$, where $S_{E}=\{x \in E:\|x\|=1\}$. It is said to be Fréchet differentiable if for each $x$ in $S_{E}$, this limit is attained uniformly for $y$ in $S_{E}$. Let $C$ be a closed convex subset of $E$ and let $T$ be a mapping of $C$ into itself. Then, for each $\varepsilon>0$, we define the set $F_{\varepsilon}(T)$ to be

$$
F_{\varepsilon}(T)=\{x \in C:\|T x-x\| \leqslant \varepsilon\} .
$$

The following lemmas were proved in [2].

LEMma 1. Let $E$ be a uniformly convex Banach space and let $C$ be a nonempty bounded closed convex subset of $E$. Then, for each $\varepsilon>0$, there exists $\delta>0$ such that

$$
\overline{c o} F_{\delta}(T) \subset F_{\varepsilon}(T)
$$

for every nonexpansive mapping $T$ of $C$ into itself.

Lemma 2. Let $E$ be a uniformly convex Banach space and let $C$ be a nonempty bounded closed convex subset of $E$. Then,

$$
\lim _{n \rightarrow \infty} \sup _{\substack{x \in C \\ T \in N(C)}}\left\|\frac{1}{n+1} \sum_{i=0}^{n} T^{i} x-T\left(\frac{1}{n+1} \sum_{i=0}^{n} T^{i} x\right)\right\|=0,
$$

where $N(C)$ denotes the set of all nonexpansive mappings of $C$ into itself. 


\section{Lemmas}

Let $C$ be a nonempty closed convex subset of a Banach space $E$. Let $S$ and $T$ be nonexpansive mappings of $C$ into itself such that $S T=T S$ and $F(S) \cap F(T) \neq \emptyset$. Now consider the following iteration scheme:

$$
x_{1}=x \in C, x_{n+1}=\alpha_{n} x_{n}+\left(1-\alpha_{n}\right) \frac{1}{n^{2}} \sum_{i, j=0}^{n-1} S^{i} T^{j} x_{n} \quad \text { for every } \quad n \in \mathbb{N},
$$

where $\left\{\alpha_{n}\right\}$ is a sequence in $[0,1]$. Then, for any $n \in \mathbb{N}$, putting

$$
T_{n} x=\alpha_{n} x+\left(1-\alpha_{n}\right) \frac{1}{n^{2}} \sum_{i, j=0}^{n-1} S^{i} T^{j} x \quad \text { for every } \quad x \in C,
$$

the mapping $T_{n}$ of $C$ into itself is also nonexpansive. In fact, let $x, y \in C$. Then, we obtain

$$
\begin{aligned}
\left\|\frac{1}{n^{2}} \sum_{i, j=0}^{n-1} S^{i} T^{j} x-\frac{1}{n^{2}} \sum_{i, j=0}^{n-1} S^{i} T^{j} y\right\| & \leqslant \frac{1}{n^{2}} \sum_{i, j=0}^{n-1}\left\|S^{i} T^{j} x-S^{i} T^{j} y\right\| \\
& \leqslant \frac{1}{n^{2}} \sum_{i, j=0}^{n-1}\|x-y\| \\
& =\|x-y\|
\end{aligned}
$$

and hence

$$
\begin{aligned}
\left\|T_{n} x-T_{n} y\right\| & =\left\|\left\{\alpha_{n} x+\left(1-\alpha_{n}\right) \frac{1}{n^{2}} \sum_{i, j=0}^{n-1} S^{i} T^{j} x\right\}-\left\{\alpha_{n} y+\left(1-\alpha_{n}\right) \frac{1}{n^{2}} \sum_{i, j=0}^{n-1} S^{i} T^{j} y\right\}\right\| \\
& \leqslant \alpha_{n}\|x-y\|+\left(1-\alpha_{n}\right)\left\|\frac{1}{n^{2}} \sum_{i, j=0}^{n-1} S^{i} T^{j} x-\frac{1}{n^{2}} \sum_{i, j=0}^{n-1} S^{i} T^{j} y\right\| \\
& \leqslant \alpha_{n}\|x-y\|+\left(1-\alpha_{n}\right)\|x-y\|=\|x-y\| .
\end{aligned}
$$

Further, we have $F(T) \cap F(S) \subset F\left(1 / n^{2} \sum_{i, j=0}^{n-1} S^{i} T^{j}\right) \subset F\left(T_{n}\right)$ for every $n \in \mathbb{N}$ and hence $F(T) \cap F(S) \subset \bigcap_{n=1}^{\infty} F\left(T_{n}\right)$

The iterates $\left\{x_{n}\right\}$ defined by (1) can be written as

$$
x_{n+1}=T_{n} T_{n-1} \cdots T_{1} x_{1} .
$$

Putting $S_{n}=T_{n} T_{n-1} \cdots T_{1}, x_{n+1}$ will be also denoted by

$$
x_{n+1}=S_{n} x_{1} .
$$

Using Lemmas 1 and 2, we can prove the following lemma which plays an important role in this paper. 
Lemma 3. Let $C$ be a nonempty bounded closed convex subset of a uniformly convex Banach space $E$. Let $S$ and $T$ be nonexpansive mappings of $C$ into itself with $S T=T S$. Then,

$$
\lim _{n \rightarrow \infty} \sup _{x \in C}\left\|\frac{1}{n^{2}} \sum_{i, j=0}^{n-1} S^{i} T^{j} x-S\left(\frac{1}{n^{2}} \sum_{i, j=0}^{n-1} S^{i} T^{j} x\right)\right\|=0
$$

and

$$
\lim _{n \rightarrow \infty} \sup _{x \in C}\left\|\frac{1}{n^{2}} \sum_{i, j=0}^{n-1} S^{i} T^{j} x-T\left(\frac{1}{n^{2}} \sum_{i, j=0}^{n-1} S^{i} T^{j} x\right)\right\|=0 .
$$

Proof: Let $\varepsilon>0$. From Lemma 1, we know that there exists $\delta>0$ such that

$$
\overline{c o} F_{\delta}(U) \subset F_{\varepsilon}(U)
$$

for every nonexpansive mapping $U$ of $C$ into itself. From Lemma 2, we also have

$$
\lim _{n \rightarrow \infty} \sup _{y \in C}\left\|\frac{1}{n} \sum_{i=0}^{n-1} S^{i} y-S\left(\frac{1}{n} \sum_{i=0}^{n-1} S^{i} y\right)\right\|=0 .
$$

Then, there exists $n_{1} \in \mathbb{N}$ such that

$$
\sup _{y \in C}\left\|\frac{1}{n} \sum_{i=0}^{n-1} S^{i} y-S\left(\frac{1}{n} \sum_{i=0}^{n-1} S^{i} y\right)\right\|<\delta
$$

for every $n \geqslant n_{1}$. Then, we obtain that

$$
\frac{1}{n} \sum_{i=0}^{n-1} S^{i} y \in F_{\delta}(S) \subset \overline{c o} F_{\delta}(S)
$$

for every $y \in C$ and $n \geqslant n_{1}$. Let $l, p \in \mathbb{N}$. Then, we have, for any $n \in \mathbb{N}$ with $n>l, p$ and $x \in C$,

$$
\begin{aligned}
\left\|\frac{1}{n^{2}} \sum_{i, j=0}^{n-1} S^{i} T^{j} x-S\left(\frac{1}{n^{2}} \sum_{i, j=0}^{n-1} S^{i} T^{j} x\right)\right\| \leqslant & \left\|\frac{1}{n^{2}} \sum_{i, j=0}^{n-1} S^{i} T^{j} x-\frac{1}{n^{2}} \sum_{i, j=0}^{n-1} S^{l+i} T^{p+j} x\right\| \\
& +\left\|\frac{1}{n^{2}} \sum_{i, j=0}^{n-1} S^{l+i} T^{p+j} x-S\left(\frac{1}{n^{2}} \sum_{i, j=0}^{n-1} S^{l+i} T^{p+j} x\right)\right\| \\
& +\left\|S\left(\frac{1}{n^{2}} \sum_{i, j=0}^{n-1} S^{l+i} T^{p+j} x\right)-S\left(\frac{1}{n^{2}} \sum_{i, j=0}^{n-1} S^{i} T^{j} x\right)\right\| \\
\leqslant & 2\left\|\frac{1}{n^{2}} \sum_{i, j=0}^{n-1} S^{i} T^{j} x-\frac{1}{n^{2}} \sum_{i, j=0}^{n-1} S^{l+i} T^{p+j} x\right\| \\
& +\left\|\frac{1}{n^{2}} \sum_{i, j=0}^{n-1} S^{l+i} T^{p+j} x-S\left(\frac{1}{n^{2}} \sum_{i, j=0}^{n-1} S^{l+i} T^{p+j} x\right)\right\|
\end{aligned}
$$


and

$$
\begin{aligned}
I & =\left\|\frac{1}{n^{2}} \sum_{i, j=0}^{n-1} S^{i} T^{j} x-\frac{1}{n^{2}} \sum_{i, j=0}^{n-1} S^{l+i} T^{p+j} x\right\| \\
& =\left\|\frac{1}{n^{2}}\left(\sum_{i=0}^{n-1} \sum_{j=0}^{p-1} S^{i} T^{j} x+\sum_{i=0}^{l-1} \sum_{j=p}^{n-1} S^{i} T^{j} x-\sum_{i=n}^{n-1+l} \sum_{j=p}^{n-1} S^{i} T^{j} x-\sum_{i=l}^{n-1+l} \sum_{j=n}^{n+n-1} S^{i} T^{j} x\right)\right\| \\
& \leqslant \frac{1}{n^{2}}\left(\sum_{i=0}^{n-1} \sum_{j=0}^{p-1}\left\|S^{i} T^{j} x\right\|+\sum_{i=0}^{l-1} \sum_{j=p}^{n-1}\left\|S^{i} T^{j} x\right\|+\sum_{i=n}^{n-1+l n-1} \sum_{j=p}^{n-1+l p+n-1}\left\|S^{i} T^{j} x\right\|+\sum_{i=l}^{n} \sum_{j=n}\left\|S^{i} T^{j} x\right\|\right) \\
& \leqslant \frac{1}{n^{2}}\{n p+l(n-p)+l(n-p)+n p\} \cdot M \\
& \leqslant \frac{2 M(l+p)}{n},
\end{aligned}
$$

where $M=\sup _{z \in C}\|z\|$. Then, there exists $n_{0} \in \mathbb{N}$ such that $n_{0}>\max \left\{n_{1}, l, p\right\}$ and $(2 M(l+p)) / n<\varepsilon$ for every $n \geqslant n_{0}$. This implies that

$$
I=\left\|\frac{1}{n^{2}} \sum_{i, j=0}^{n-1} S^{i} T^{j} x-\frac{1}{n^{2}} \sum_{i, j=0}^{n-1} S^{l+i} T^{p+j} x\right\|<\varepsilon \quad \text { for every } \quad x \in C .
$$

Next, we prove that $1 / n^{2} \sum_{i, j=0}^{n-1} S^{m+i} T^{q+j} x \in \overline{c o} F_{\delta}(S)$ for every $x \in C, m, q \in \mathbb{N}$ and $n \geqslant n_{1}$. If not, we have that for some $m, q \in \mathbb{N}, n \geqslant n_{1}$ and $x \in C$

$$
\frac{1}{n^{2}} \sum_{i, j=0}^{n-1} S^{m+i} T^{q+j} x \notin \overline{c o} F_{\delta}(S) \text {. }
$$

From the separation theorem, there exists $y_{1}^{*} \in E^{*}$ such that

$$
\left\langle\frac{1}{n^{2}} \sum_{i, j=0}^{n-1} S^{m+i} T^{q+j} x, y_{1}^{*}\right\rangle\left\langle\inf \left\{\left\langle z, y_{1}^{*}\right\rangle: z \in \overline{c o} F_{\delta}(S)\right\} .\right.
$$

Then, from (5), we obtain

$$
\begin{aligned}
\inf \left\{\left\langle z, y_{1}^{*}\right\rangle: z \in \overline{c o} F_{\delta}(S)\right\} & \leqslant \inf \left\{\left\langle\frac{1}{n} \sum_{i=0}^{n-1} S^{i} x, y_{1}^{*}\right\rangle: x \in C\right\} \\
& \leqslant\left\langle\frac{1}{n} \sum_{i=0}^{n-1} S^{i} y, y_{1}^{*}\right\rangle
\end{aligned}
$$

for all $y \in C$ and $n \geqslant n_{1}$. Then, we have that for any $j \in\{0,1,2, \ldots, n-1\}$

$$
\inf \left\{\left\langle z, y_{1}^{*}\right\rangle: z \in \overline{c o} F_{\delta}(S)\right\} \leqslant\left\langle\frac{1}{n} \sum_{i=0}^{n-1} S^{i}\left(S^{m} T^{q+j} x\right), y_{1}^{*}\right\rangle
$$


and hence

$$
\inf \left\{\left\langle z, y_{1}^{*}\right\rangle: z \in \overline{c o} F_{\delta}(S)\right\} \leqslant \frac{1}{n} \sum_{j=0}^{n-1}\left\langle\frac{1}{n} \sum_{i=0}^{n-1} S^{i}\left(S^{m} T^{q+j} x\right), y_{1}^{*}\right\rangle
$$

Therefore, from (6),

$$
\begin{aligned}
\inf \left\{\left\langle z, y_{1}^{*}\right\rangle: z \in \overline{c o} F_{\delta}(S)\right\} & \leqslant \frac{1}{n} \sum_{j=0}^{n-1}\left\langle\frac{1}{n} \sum_{i=0}^{n-1} S^{i}\left(S^{m} T^{q+j} x\right), y_{1}^{*}\right\rangle \\
& <\inf \left\{\left\langle z, y_{1}^{*}\right\rangle: z \in \overline{c o} F_{\delta}(S)\right\}
\end{aligned}
$$

This is a contradiction. Hence, from (4), we have

$$
\frac{1}{n^{2}} \sum_{i, j=0}^{n-1} S^{m+i} T^{q+j} x \in \overline{c o} F_{\delta}(S) \subset F_{\varepsilon}(S)
$$

for every $m, q \in \mathbb{N}, x \in C$ and $n \geqslant n_{1}$. Then, it follows from (7) that

$$
\sup _{\substack{x \in C \\ m, q \in \mathbb{N}}}\left\|\frac{1}{n^{2}} \sum_{i, j=0}^{n-1} S^{m+i} T^{q+j} x-S\left(\frac{1}{n^{2}} \sum_{i, j=0}^{n-1} S^{m+i} T^{q+j} x\right)\right\|<\varepsilon
$$

for every $n \geqslant n_{1}$. Hence, we obtain

$$
\left\|\frac{1}{n^{2}} \sum_{i, j=0}^{n-1} S^{i} T^{j} x-S\left(\frac{1}{n^{2}} \sum_{i, j=0}^{n-1} S^{i} T^{j} x\right)\right\| \leqslant 2 \varepsilon+\varepsilon=3 \varepsilon
$$

for every $x \in C$ and $n \geqslant n_{0}$. Therefore,

$$
\lim _{n \rightarrow \infty} \sup _{x \in C}\left\|\frac{1}{n^{2}} \sum_{i, j=0}^{n-1} S^{i} T^{j} x-S\left(\frac{1}{n^{2}} \sum_{i, j=0}^{n-1} S^{i} T^{j} x\right)\right\|=0 .
$$

Similarly, we have

$$
\lim _{n \rightarrow \infty} \sup _{x \in C}\left\|\frac{1}{n^{2}} \sum_{i, j=0}^{n-1} S^{i} T^{j} x-T\left(\frac{1}{n^{2}} \sum_{i, j=0}^{n-1} S^{i} T^{j} x\right)\right\|=0 .
$$

The following lemma was essentially proved by Reich [7] (see also [8]).

LEMMA 4. Let $C$ be a nonempty closed convex subset of a uniformly convex Banach space $E$ with a Fréchet differentiable norm and let $\left\{T_{1}, T_{2}, T_{3}, \ldots\right\}$ be a sequence of nonexpansive mappings of $C$ into itself such that $\bigcap_{n=1}^{\infty} F\left(T_{n}\right)$ is nonempty. Let $x \in C$ and set $S_{n}=T_{n} T_{n-1} \cdots T_{1}$ for every $n \in \mathbb{N}$. Then, the set $\bigcap_{n=1}^{\infty} \overline{c o}\left\{S_{m} x: m \geqslant n\right\} \cap U$ consists of at most one point, where $U=\bigcap_{n=1}^{\infty} F\left(T_{n}\right)$. 
We also have the following lemma.

Lemma 5. Let $E$ be a Banach space and let $C$ be a nonempty closed convex subset of $E$. Let $S$ and $T$ be nonexpansive mappings of $C$ into itself such that $S T=T S$ and $F(S) \cap F(T) \neq \emptyset$. Suppose $x_{1}=x \in C$ and $\left\{x_{n}\right\}$ is given by

$$
x_{n+1}=\alpha_{n} x_{n}+\left(1-\alpha_{n}\right) \frac{1}{n^{2}} \sum_{i, j=0}^{n-1} S^{i} T^{j} x_{n} \quad \text { for every } n \in \mathbb{N},
$$

where $\left\{\alpha_{n}\right\}$ is a sequence in $[0,1]$. Let $w$ be a common fixed point of $T$ and $S$. Then, $\lim _{n \rightarrow \infty}\left\|x_{n}-w\right\|$ exists.

Proof: Let $w$ be a common fixed point of $T$ and $S$. Then, we have

$$
\begin{aligned}
\left\|x_{n+1}-w\right\| & =\left\|\alpha_{n} x_{n}+\left(1-\alpha_{n}\right) \frac{1}{n^{2}} \sum_{i, j=0}^{n-1} S^{i} T^{j} x_{n}-w\right\| \\
& \leqslant \alpha_{n}\left\|x_{n}-w\right\|+\left(1-\alpha_{n}\right)\left\|\frac{1}{n^{2}} \sum_{i, j=0}^{n-1} S^{i} T^{j} x_{n}-w\right\| \\
& \leqslant \alpha_{n}\left\|x_{n}-w\right\|+\left(1-\alpha_{n}\right)\left\|x_{n}-w\right\| \\
& =\left\|x_{n}-w\right\|
\end{aligned}
$$

and hence $\lim _{n \rightarrow \infty}\left\|x_{n}-w\right\|$ exists.

The following lemma was proved in [9].

LEMMA 6. Let $p>1$ and $r>0$ be two fixed real numbers. Let $E$ be a uniformly convex Banach space. Then, there is a continuous, strictly increasing and convex function $g:[0, \infty) \rightarrow[0, \infty)$ such that $g(0)=0$ and

$$
\|\lambda x+(1-\lambda) y\|^{p} \leqslant \lambda\|x\|^{p}+(1-\lambda)\|y\|^{p}-\left\{\lambda^{p}(1-\lambda)+\lambda(1-\lambda)^{p}\right\} g(\|x-y\|)
$$

for all $x, y \in B_{r}$ and $\lambda$ with $0 \leqslant \lambda \leqslant 1$, where $B_{r}=\{x \in E:\|x\| \leqslant r\}$.

Using Lemmas 3 and 6 , we can show the following lemma which is essential to prove the weak convergence theorem (Theorem 1).

Lemma 7. Let $C$ be a nonempty closed convex subset of a uniformly convex Banach space $E$. Let $S$ and $T$ be nonexpansive mappings of $C$ into itself such that $S T=T S$ and $F(S) \cap F(T) \neq \emptyset$. Suppose $x_{1}=x \in C$ and $\left\{x_{n}\right\}$ is given by

$$
x_{n+1}=\alpha_{n} x_{n}+\left(1-\alpha_{n}\right) \frac{1}{n^{2}} \sum_{i, j=0}^{n-1} S^{i} T^{j} x_{n} \quad \text { for every } n \in \mathbb{N},
$$

where $0 \leqslant \alpha_{n} \leqslant a$ for some $a$ with $0<a<1$. Then,

$$
\lim _{n}\left\|T x_{n}-x_{n}\right\|=\lim _{n}\left\|S x_{n}-x_{n}\right\|=0 .
$$

In particular, $x_{n_{i}} \rightarrow y_{0}$ implies $y_{0} \in F(T) \cap F(S)$. 
Proof: For $x \in C$ and $f \in F(T) \cap F(S)$, put $r=\|x-f\|$ and set $X=\{u \in E$ : $\|u-f\| \leqslant r\} \cap C$. Then, $X$ is a nonempty bounded closed convex subset of $C$ which is $T, S$-invariant and contains $x_{1}=x$. So, without loss of generality, we may assume that $C$ is bounded. Let $w$ be a common fixed point of $T$ and $S$. Then, from Lemma 6 , there exists a continuous, strictly increasing and convex function $g:[0, \infty) \rightarrow[0, \infty)$ such that $g(0)=0$ and

$$
\begin{aligned}
\left\|x_{n+1}-w\right\|^{2} & =\left\|\alpha_{n}\left(x_{n}-w\right)+\left(1-\alpha_{n}\right)\left(\frac{1}{n^{2}} \sum_{i, j=0}^{n-1} S^{i} T^{j} x_{n}-w\right)\right\|^{2} \\
& \leqslant \alpha_{n}\left\|x_{n}-w\right\|^{2}+\left(1-\alpha_{n}\right)\left\|\frac{1}{n^{2}} \sum_{i, j=0}^{n-1} S^{i} T^{j} x_{n}-w\right\|^{2} \\
& -\alpha_{n}\left(1-\alpha_{n}\right) g\left(\| \frac{1}{n^{2}} \sum_{i, j=0}^{n-1} S^{i} T^{j} x_{n}-x_{n} \mid i\right)
\end{aligned}
$$

for all $n \in \mathbb{N}$. Then, since $\alpha_{n} \leqslant a$, we have

$$
\begin{aligned}
\alpha_{n}(1-a) g & \left(\left\|\frac{1}{n^{2}} \sum_{i, j=0}^{n-1} S^{i} T^{j} x_{n}-x_{n}\right\|\right) \\
& \leqslant \alpha_{n}\left(1-\alpha_{n}\right) g\left(\left\|\frac{1}{n^{2}} \sum_{i, j=0}^{n-1} S^{i} T^{j} x_{n}-x_{n}\right\|\right) \\
& \leqq \alpha_{n}\left\|x_{n}-w\right\|^{2}+\left(1-\alpha_{n}\right)\left\|\frac{1}{n^{2}} \sum_{i, j=0}^{n-1} S^{i} T^{j} x_{n}-w\right\|^{2}-\left\|x_{n+1}-w\right\|^{2} \\
& \leqslant \alpha_{n}\left\|x_{n}-w\right\|^{2}+\left(1-\alpha_{n}\right)\left\|x_{n}-w\right\|^{2}-\left\|x_{n+1}-w\right\|^{2} \\
& =\left\|x_{n}-w\right\|^{2}-\left\|x_{n+1}-w\right\|^{2} .
\end{aligned}
$$

So, from Lemma 5 , we obtain

$$
\lim _{n \rightarrow \infty} \alpha_{n} g\left(\left\|\frac{1}{n^{2}} \sum_{i, j=0}^{n-1} S S^{i} T^{j} x_{n}-x_{n}\right\|\right)=0 .
$$

Since $g$ is continuous, strictly increasing and satisfies $g(0)=0$, we have

$$
\lim _{n \rightarrow \infty} \alpha_{n}\left\|\frac{1}{n^{2}} \sum_{i, j=0}^{n-1} S^{i} T^{j} x_{n}-x_{n}\right\|=0 .
$$

It follows from the definition of $\left\{x_{n}\right\}$ that

$$
x_{n+1}-\frac{1}{n^{2}} \sum_{i, j=0}^{n-1} S^{i} T^{j} x_{n}=\alpha_{n}\left(x_{n}-\frac{1}{n^{2}} \sum_{i, j=0}^{n-1} S^{i} T^{j} x_{n}\right) .
$$


Since

$$
\begin{aligned}
\| T x_{n+1}- & x_{n+1} \| \\
\leqslant & \left\|T x_{n+1}-T\left(\frac{1}{n^{2}} \sum_{i, j=0}^{n-1} S^{i} T^{j} x_{n}\right)\right\| \\
& +\left\|T\left(\frac{1}{n^{2}} \sum_{i, j=0}^{n-1} S^{i} T^{j} x_{n}\right)-\frac{1}{n^{2}} \sum_{i, j=0}^{n-1} S^{i} T^{j} x_{n}\right\|+\left\|\frac{1}{n^{2}} \sum_{i, j=0}^{n-1} S^{i} T^{j} x_{n}-x_{n+1}\right\| \\
\leqslant & 2\left\|\frac{1}{n^{2}} \sum_{i, j=0}^{n-1} S^{i} T^{j} x_{n}-x_{n+1}\right\|+\left\|T\left(\frac{1}{n^{2}} \sum_{i, j=0}^{n-1} S^{i} T^{j} x_{n}\right)-\frac{1}{n^{2}} \sum_{i, j=0}^{n-1} S^{i} T^{j} x_{n}\right\| \\
= & 2 \alpha_{n}\left\|x_{n}-\frac{1}{n^{2}} \sum_{i, j=0}^{n-1} S^{i} T^{j} x_{n}\right\|+\left\|T\left(\frac{1}{n^{2}} \sum_{i, j=0}^{n-1} S^{i} T^{j} x_{n}\right)-\frac{1}{n^{2}} \sum_{i, j=0}^{n-1} S^{i} T^{j} x_{n}\right\|,
\end{aligned}
$$

from (8) and Lemma 3, we have

$$
\lim _{n \rightarrow \infty}\left\|T x_{n}-x_{n}\right\|=0
$$

Similarly, we have

$$
\lim _{n \rightarrow \infty}\left\|S x_{n}-x_{n}\right\|=0 .
$$

Assume $x_{n_{i}} \rightarrow y_{0}$. Then, since $I-T$ and $I-S$ are demiclosed by $[\mathbf{1}]$, we obtain that $y_{0}$ is a common fixed point of $T$ and $S$.

\section{Main Result}

We shall prove the main result in this paper.

THEOREM 1. Let $E$ be a uniformly convex Banach space which satisfies Opial's condition or whose norm is Fréchet differentiable. Let $C$ be a nonempty closed convex subset of $E$. Let $S$ and $T$ be nonexpansive mappings of $C$ into itself such that $S T=T S$ and $F(S) \cap F(T) \neq \emptyset$. Suppose $x_{1}=x \in C$ and $\left\{x_{n}\right\}$ is given by

$$
x_{n+1}=\alpha_{n} x_{n}+\left(1-\alpha_{n}\right) \frac{1}{n^{2}} \sum_{i, j=0}^{n-1} S^{i} T^{j} x_{n} \quad \text { for every } n \in \mathbb{N},
$$

where $\left\{\alpha_{n}\right\}$ is a sequence in $[0,1]$. If $\left\{\alpha_{n}\right\}$ is chosen so that $\alpha_{n} \in[0, a]$ for some a with $0<a<1$, then $\left\{x_{n}\right\}$ converges weakly to a common fixed point $z_{0}$ of $T$ and $S$.

Proof: Let $x \in C$. We first assume that $E$ satisfies Opial's condition. Let $w$ be a common fixed point of $T$ and $S$. Then, from Lemma $5, \lim _{n \rightarrow \infty}\left\|x_{n}-w\right\|$ exists. As in the proof of Lemma 7, we may assume that $C$ is bounded. Since $E$ is reflexive, $\left\{x_{n}\right\}$ must contain a subsequence which converges weakly to a point in $C$. So, let $\left\{x_{n_{i}}\right\}$ and $\left\{x_{n_{j}}\right\}$ be two subsequences of $\left\{x_{n}\right\}$ such that $x_{n_{i}} \rightarrow z_{1}$ and $x_{n_{j}} \rightarrow z_{2}$. Then, from Lemma 7 , 
we have that $z_{1}$ and $z_{2}$ are common fixed points of $T$ and $S$. Next, we show $z_{1}=z_{2}$. If not, from Opial's condition,

$$
\begin{aligned}
\lim _{n \rightarrow \infty}\left\|x_{n}-z_{1}\right\| & =\lim _{i \rightarrow \infty}\left\|x_{n_{i}}-z_{1}\right\| \\
& <\lim _{i \rightarrow \infty}\left\|x_{n_{i}}-z_{2}\right\|=\lim _{n \rightarrow \infty}\left\|x_{n}-z_{2}\right\|=\lim _{j \rightarrow \infty}\left\|x_{n_{j}}-z_{2}\right\| \\
& <\lim _{j \rightarrow \infty}\left\|x_{n_{j}}-z_{1}\right\|=\lim _{n \rightarrow \infty}\left\|x_{n}-z_{1}\right\| .
\end{aligned}
$$

This is a contradiction. Hence, we obtain $x_{n} \rightarrow y_{0} \in F(T) \cap F(S)$.

Next, we assume that $E$ has a Fréchet differentiable norm. As in the proof of Lemma 7 , we may assume that $C$ is bounded. So, there exists a subsequence $\left\{x_{n_{i}}\right\}$ of $\left\{x_{n}\right\}$ such that $x_{n_{i}} \rightarrow y_{0}$. Then, from Lemma 7, we obtain $y_{0} \in F(T) \cap F(S)$. Putting $T_{n} y=\alpha_{n} y+\left(1-\alpha_{n}\right) 1 / n^{2} \sum_{i, j=0}^{n-1} S^{i} T^{j} y$ and $S_{n} y=T_{n} T_{n-1} T_{n-2} \cdots T_{1} y$ for all $n \in \mathbb{N}$ and $y \in C$, from (3), we have $y_{0} \in \bigcap_{n=1}^{\infty} \overline{c o}\left\{S_{m} x: m \geqslant n\right\}$. Hence, we have

$$
y_{0} \in \bigcap_{n=1}^{\infty} \overline{c o}\left\{S_{m} x: m \geqslant n\right\} \cap F(T) \cap F(S) \subset \bigcap_{n=1}^{\infty} \overline{c o}\left\{S_{m} x: m \geqslant n\right\} \cap \bigcap_{n=1}^{\infty} F\left(T_{n}\right)
$$

So, from Lemma 4, we have

$$
\left\{y_{0}\right\}=\bigcap_{n=1}^{\infty} \overline{c o}\left\{S_{m} x: m \geqslant n\right\} \cap \bigcap_{n=1}^{\infty} F\left(T_{n}\right)
$$

Hence, we obtain $x_{n} \rightarrow y_{0} \in F(T) \cap F(S)$.

\section{REFERENCES}

[1] F.E. Browder, 'Nonlinear operators and nonlinear equations of evolution in Banach spaces', Amer. Math. Soc. 18 (1976).

[2] R.E. Bruck, 'On the convex approximation property and the asymptotic behavior of nonlinear contractions in Banach spaces', Israel J. Math. 38 (1981), 304-314.

[3] D. Van Dulst, 'Equivalent norms and the fixed point property for nonexpansive mappings', J. London. Math. Soc. 25 (1982), 139-144.

[4] J.P. Gossez and E. Lami Dozo, 'Some geometric properties related to the fixed point theory for nonexpansive mappings', Pacific. J. Math. 40 (1972), 565-573.

[5] W.R. Mann, 'Mean value methods in iteration', Proc. Amer. Math. Soc. 4 (1953), 506-510.

[6] Z. Opial, 'Weak convergence of the sequence of successive approximations for nonexpansive mappings', Bull. Amer. Math. Soc. 73 (1967), 591-597. 
[7] S. Reich, 'Weak convergence theorems for nonexpansive mappings in Banach spaces', $J$. Math. Anal. Appl. 67 (1979), 274-276.

[8] W. Takahashi and G. E. Kim, 'Approximating fixed points of nonexpansive mappings in Banach spaces', Mathematica Japonica (to appear).

[9] H.K. Xu, 'Inequalities in Banach spaces with applications', Nonlinear Anal. 16 (1991), 1127-1138.

Department of Mathematical and Computing Sciences

Tokyo Institute of Technology

O-okayama, Meguro-ku

Tokyo 152, Japan

e-mail: atsusiba@is.titech.ac.jp

wataru@is.titech.ac.jp 